\title{
Servicio enfermero ofertado en una unidad de hospitalización de Nefrología
}

\author{
Ma Ángeles Cidoncha Moreno* \\ Ma Soledad Díez de Baldeón Herrero**
}

*Supervisora Unidad Nefrología y Hemodiálisis

**Enfermera Diálisis Peritoneal

Hospital Santiago Apóstol de Vitoria (Álava)

\section{RESUMEN}

La aportación de la disciplina enfermera se concreta en la prestación de cuidados, y el proceso de enfermería permite reconocer la aportación de las enfermeras a los resultados de salud del paciente.

En el Servicio Vasco de Salud (O sakidetza) se trabaja con el programa informático Zaineri, que recoge bajo soporte informático todos los cuidados y actividades que la enfermera realiza en la práctica diaria. Es fundamental que en las unidades se analice qué servicio estamos ofertando a nuestros clientes. Por ello el objetivo de este trabajo es describir el trabajo que realizan las enfermeras de Nefrología, a través del análisis de las necesidades alteradas de los pacientes ingresados y de los diagnósticos de enfermería planteados durante el año 2002.

Material y método: estudio descriptivo de los planes de cuidados de los pacientes ingresados en la unidad entre el 1 de Enero de 2002 y el 31 de Diciembre de 2002.

Resultados: del total de necesidades planteadas, el $65,30 \%$ pertenecen al área independiente de Enfermería mientras un $34,70 \%$ pertenecen al área interdependiente. Los diagnósticos derivados de Necesidades Humanas corresponden al 56,63\% sobre el total, mientras aquellos diagnósticos (complicaciones) derivados de los sistemas, tienen un porcentaje del $43,37 \%$.

\section{Correspondencia: \\ M. Ángeles Cidoncha \\ U nidad de Hemodiálisis \\ Hospital Santiago A póstol \\ C/ Olaguibel no 29 \\ 01004 Vitoria (Álava) \\ mcidoncha@hsan.osakidetza.net}

\section{PALABRAS CLAVE: SERVICIO ENFERMERO \\ ZAINERI \\ DIAGNÓSTICOS ENFERMEROS \\ NEFROLOGÍA \\ CUIDADOS}

\section{NURSING SERVICES OFFERED IN A HOSPITAL UNIT FOR NEPHROLOGY}

Nursing skills are offering a number of services to the patient, including its care. Nursing processes allow the identification of results on the patient's health.

In the Vasc Service of Health (O sakidetza) we work with the programme Zaineri that collects all processes done by nurses in their daily activities.

Is is important to analyze what kind of service we are offering to our clients, therefore, the aim of this work is to describe the work done by nurses of nephrology through the analysis of the patient's needs and nursing diagnosis during 2002.

Material and methods: descriptive study of the care plan for patients from jan 1 st 2002 to dec $31^{\text {st }}$ 2002.

Results: from all needs analyze, $65 \%$ belong to the nurse independent area, while $34 \%$ belong to the interdependent area. Diagnosis obtained from human needs are $57 \%$ while those obtained from systems are $43 \%$.

$\begin{array}{ll}\text { KEY WORDS: } & \text { NURSING SERVICE } \\ & \text { ZAINERI } \\ & \text { NURSING DIAGNOSIS } \\ & \text { NEPHROLOGY } \\ & \text { CARE }\end{array}$




\section{INTRODUCCIÓN}

En el hospital confluyen muchos flujos de trabajo que persiguen objetivos concretos. La necesidad de cuidados en todos los establecimientos sanitarios hace indispensable la presencia de los profesionales de enfermería para intervenir en todos los procesos que en ellos se desarrollan.

Durante mucho tiempo se ha entendido la actividad enfermera como auxiliar, secundaria de la actividad médica de curar o sanar, pero más recientemente, se tiene conciencia que la Enfermería desarrolla funciones y actividades que le son propias y exclusivas, concretándose la aportación de la disciplina enfermera en la prestación de cuidados.

Existe consenso en que la utilización del diagnóstico enfermero es piedra angular para el desarrollo profesional, ya que nos ofrece un idioma y unas fórmulas que permiten entendernos entre nosotros, describiendo uniformemente lo que hacemos, comunicarlo y dejar constancia de ello en los diferentes ámbitos de la atención de la salud.

El proceso de enfermería es lo que permite reconocer la aportación de las enfermeras a los resultados de salud del paciente. Este proceso se manifiesta en el plan de cuidados, documentación que recoge la planificación de los cuidados de un paciente concreto ante una situación determinada. El plan de cuidados es indispensable para conocer la contribución específica de las enfermeras al cuidado de la salud.

En el Servicio Vasco de Salud (Osakidetza) se trabaja con el programa informático Zaineri, que recoge bajo soporte informático todos los cuidados y actividades que la enfermera realiza en la práctica diaria. Se apoya por un lado en el modelo de Enfermería de Virginia Henderson que hace referencia a los cuidados relacionados con el área independiente de Enfermería, basada en $\mathrm{Ne}$ cesidades Humanas, y por otro lado, en un modelo biológico (basado en sistemas y aparatos), que refleja las actividades relacionadas con el área interdependiente, de colaboración, cuya misión principal es la vigilancia de las manifestaciones de determinados signos y síntomas para la prevención de posibles complicaciones.

Estructuralmente el programa Zaineri tiene establecidos 5 niveles informáticos:
1. Necesidades/Sistemas

2. Problemas/Complicaciones

3. Con qué están relacionados

4. Objetivos

5. Actividades

Es fundamental que en las unidades se analice qué servicio estamos ofertando a nuestros clientes, ya que a partir de los planes de cuidados estandarizados e individualizados estamos ofertando unas determinadas actividades cuidadoras encaminadas a satisfacer las necesidades de las personas en relación a su salud, lo que dará a la unidad un carácter especial y distintivo. Para profundizar en este tema se realizó este trabajo para intentar responder a la pregunta de ¿Q ué servicio ofertamos a los pacientes de Nefrología?

\section{OBJ ETIVO}

Describir el trabajo que realizan las enfermeras de $\mathrm{Ne}$ frología, a través del análisis de las necesidades alteradas de los pacientes ingresados y de los diagnósticos de enfermería planteados durante el año 2002.

\section{MATERIAL Y MÉTODO}

- Estudio observacional descriptivo de los planes de cuidados de los pacientes ingresados en la unidad, investigando las necesidades alteradas y los diagnósticos enfermeros planteados

- Periodo de estudio: del 1 de Enero de 2002 a 31 de Diciembre de 2002

- Ámbito de estudio: Unidad de hospitalización de Nefrología

- Sujetos de estudio: se incluyó a todos los pacientes de la especialidad de Nefrología dados de alta en la unidad durante el periodo de estudio

- Los datos fueron extraídos a través del programa informático de cuidados "ZAINERI" apoyado en el modelo de Virginia Henderson, en un modelo Biomédico y en el Proceso de Atención de Enfermería como método de resolución de problemas en los que intervienen las enfermeras

- El análisis estadístico se efectuó a través del paquete informático de Microsoft Excel expresándose como porcentajes 


\section{RESULTADOS}

Las necesidades que aparecen más alteradas son las de A prendizaje, Integridad de piel y mucosas y Seguridad Física y Psíquica. En cuanto a los Sistemas, es el Cardiovascular en el que se recogen más incidencias (tabla 1). Del total de necesidades planteadas, el $65,30 \%$ pertenecen al área independiente de Enfermería, mientras un $34,70 \%$ pertenecen al área interdependiente 0 de colaboración con los médicos.

\begin{tabular}{|l|c|c|}
\hline NECESIDADES & $\begin{array}{c}\text { \% pacientes con esa } \\
\text { Necesidad o Sistema }\end{array}$ & $\begin{array}{c}\text { \% sobre el total } \\
\text { de Necesidades } 0 \\
\text { Sistemas } \\
\mathrm{N}=389\end{array}$ \\
\hline A cogida & $100 \%$ & $10,28 \%$ \\
\hline N. A prendizaje & $97,50 \%$ & $10,03 \%$ \\
\hline N. Eliminación & $35 \%$ & $3,60 \%$ \\
\hline N. Movilidad/ Actividad & $80 \%$ & $8,23 \%$ \\
\hline N. Nutrición & $90 \%$ & $9,25 \%$ \\
\hline N. Oxigenación & $12,50 \%$ & $1,29 \%$ \\
\hline N. Piel y Mucosas & $97,50 \%$ & $10,03 \%$ \\
\hline N. Reposo/Sueño & $15 \%$ & $1,54 \%$ \\
\hline N. Seguridad Física y Psiquíca & $97,50 \%$ & $10,03 \%$ \\
\hline N. Termorregulación & $10 \%$ & $1,03 \%$ \\
\hline Orden Médica & $85 \%$ & $8,74 \%$ \\
\hline S. Cardiovascular & $97,50 \%$ & $10,03 \%$ \\
\hline S. Digestivo/ Metabólico & $27,50 \%$ & $2,83 \%$ \\
\hline S. Genito/U rinario & $65 \%$ & $6,68 \%$ \\
\hline S. M úsculo/ Esquelético & $7,5 \%$ & $0,77 \%$ \\
\hline S. Neurológico & $20 \%$ & $2,06 \%$ \\
\hline S. Respiratorio & $22,50 \%$ & $2,31 \%$ \\
\hline S. Tegumentario & $12,50 \%$ & $1,29 \%$ \\
\hline
\end{tabular}

Tabla 1. Alteraciones en Necesidades y Sistemas de la muestra a estudio.

Los diagnósticos derivados de Necesidades corresponden al 56,63\% del total, mientras las complicaciones derivadas de los sistemas, tienen un porcentaje del $43,37 \%$.

Dentro de los diagnósticos derivados de Necesidades, los que recogen un mayor porcentaje son (tabla 2):

- Riesgo de Ulcera por presión (alto, bajo y sin riesgo).

- Riesgo de Accidentes (medio, bajo y sin riesgo).

- Déficit de conocimientos relacionado con situación de salud actual y otros.
- Intolerancia a la actividad relacionado con debilidad y cansancio, desequilibrio aporte/ demanda y otros

\begin{tabular}{|c|c|}
\hline DIAGNÓSTICOS & $\begin{array}{r}\% \\
N=282\end{array}$ \\
\hline Riesgo de UPP r/c alto, bajo y sin riesgo & $12,77 \%$ \\
\hline Riesgo de Accidente r/ c medio, bajo y sin riesgo & $11,70 \%$ \\
\hline $\begin{array}{l}\text { Déficit de conocimientos r/c situación de salud actual } \\
\text { y otros }\end{array}$ & $9,57 \%$ \\
\hline $\begin{array}{l}\text { Intolerancia a la actividad r/ c debilidad/ cansancio, } \\
\text { desequilibrio aporte/ demanda y otros }\end{array}$ & $9,22 \%$ \\
\hline $\begin{array}{l}\text { Temor r/ c amenaza o cambio en estado salud, evolución } \\
\text { del proceso y sentimiento de muerte }\end{array}$ & $8,87 \%$ \\
\hline Riesgo alteración en la nutrición r/ c cansancio y otros & $8,51 \%$ \\
\hline $\begin{array}{l}\text { Déficit autocuidado: higiene y vestido r/ c proceso de } \\
\text { adaptación }\end{array}$ & $7,80 \%$ \\
\hline Deterioro integridad cutánea r/ c prurito & $7,45 \%$ \\
\hline $\begin{array}{l}\text { Riesgo déficit autocuidado: eliminación r/ c proceso } \\
\text { de adaptación y otros }\end{array}$ & $2,84 \%$ \\
\hline $\begin{array}{l}\text { Riesgo déficit autocuidado: alimentación r/ c proceso } \\
\text { de adaptación y otros }\end{array}$ & $2,48 \%$ \\
\hline $\begin{array}{l}\text { Riesgo déficit autocuidado: higiene y vestido r/ c proceso } \\
\text { de adaptación y otros }\end{array}$ & $2,48 \%$ \\
\hline $\begin{array}{l}\text { Riesgo estreñimiento r/c disminución nivel de actividad, } \\
\text { inhibición reflejo para defecar y otros }\end{array}$ & $2,48 \%$ \\
\hline Ulcera por presión r/ c presión y otros & $2,13 \%$ \\
\hline $\begin{array}{l}\text { Alteración mantenimiento de la salud } r / c \text { falta de } \\
\text { habilidad y otros }\end{array}$ & $2,13 \%$ \\
\hline $\begin{array}{l}\text { Riesgo alteración patrón de sueño r/ c cambios del } \\
\text { entorno, diseña nocturna, inactividad y otros }\end{array}$ & $1,42 \%$ \\
\hline $\begin{array}{l}\text { Riesgo limpieza ineficaz vías aéreas r/ c dificultad } \\
\text { expansión torácica, disminución de energía, reflejo } \\
\text { tusígeno ineficaz }\end{array}$ & $1,42 \%$ \\
\hline Termorregulación ineficaz r/ c otros & $1,42 \%$ \\
\hline $\begin{array}{l}\text { Déficit autocuidado: eliminación r/ c proceso de } \\
\text { adaptación y otros }\end{array}$ & $1,06 \%$ \\
\hline $\begin{array}{l}\text { Alteración de mucosa oral r/ c patrón higiene ineficaz } \\
\text { y otros }\end{array}$ & $0,71 \%$ \\
\hline $\begin{array}{l}\text { Alteración patrón de sueño } \mathrm{r} / \mathrm{c} \text { inactividad y otros } \\
\text { Deterioro movilidad física } \mathrm{r} / \mathrm{c} \text { deterioro } \\
\text { musculoesquelético y otros }\end{array}$ & $\begin{array}{l}0,71 \% \\
0,71 \%\end{array}$ \\
\hline Riesgo intolerancia a la actividad r/ c otros & $0,71 \%$ \\
\hline $\begin{array}{l}\text { Limpieza ineficaz vías aéreas r/ c dificultad expansión } \\
\text { torácica }\end{array}$ & $0,35 \%$ \\
\hline $\begin{array}{l}\text { Alteración procesos pensamiento r/ c incapacidad para } \\
\text { diferenciar realidad y creencias }\end{array}$ & $0,35 \%$ \\
\hline $\begin{array}{l}\text { Alteración sensoperceptiva (alucinaciones/ ilusiones) } \\
\text { r/ c trastornos recepción-transmisión y/ o integridad } \\
\text { sensitiva }\end{array}$ & $0,35 \%$ \\
\hline Baja autoestima situacional r/ c crisis situacionales & $0,35 \%$ \\
\hline
\end{tabular}

Tabla 2. Diagnósticos derivados de Necesidades. 
Si se analizan todos los diagnósticos relacionados con Necesidades se observa que el $46,81 \%$ son diagnósticos potenciales o de riesgo mientras un $53,19 \%$ son diagnósticos reales.

Dentro de los Sistemas, los diagnósticos de vigilancia de complicaciones de flebitis, desequilibrio hidroelectrolítico, hipertensión y oliguria son los que mayores porcentajes recogen (tabla 3).

\begin{tabular}{|c|c|}
\hline DIAGNÓSTICOS & $\begin{array}{r}\% \\
\mathbf{N}=\mathbf{2 1 6}\end{array}$ \\
\hline C: flebitis & $12,50 \%$ \\
\hline C: desequilibrio hidroelectrolítico & $11,11 \%$ \\
\hline C: hipertensión & $11,11 \%$ \\
\hline C: oliguria & $10,19 \%$ \\
\hline C: exceso volumen de líquidos & $9,72 \%$ \\
\hline C: hemorragia & $8,33 \%$ \\
\hline C: secundaria a FAVI & $6,02 \%$ \\
\hline C: infección & $5,09 \%$ \\
\hline C: dolor & $4,63 \%$ \\
\hline C: hipoxia & $3,24 \%$ \\
\hline C: hipoglucemia & $2,31 \%$ \\
\hline C: hiperglucemia & $2,31 \%$ \\
\hline C: hipotensión & $1,85 \%$ \\
\hline C: reacción transfusional & $1,39 \%$ \\
\hline C: arritmia & $0,93 \%$ \\
\hline C: íleo paralítico & $0,93 \%$ \\
\hline C: dolor precordial & $0,46 \%$ \\
\hline C: angor & $0,46 \%$ \\
\hline C: ascitis & $0,46 \%$ \\
\hline C: broncoaspiración & $0,46 \%$ \\
\hline C: desnutrición & $0,46 \%$ \\
\hline C: diarreas & $0,46 \%$ \\
\hline C: efectos secundarios anestesia & $0,46 \%$ \\
\hline C: efectos secundarios medicación psiquiátrica & $0,46 \%$ \\
\hline C: embolismo & $0,46 \%$ \\
\hline C: encefalopatía & $0,46 \%$ \\
\hline C: estasis venoso & $0,46 \%$ \\
\hline C: estomas & $0,46 \%$ \\
\hline C: hipercapnia & $0,46 \%$ \\
\hline C: obstrucción vascular & $0,46 \%$ \\
\hline C: repetición ACV & $0,46 \%$ \\
\hline C: retención urinaria & $0,46 \%$ \\
\hline C: trombosis venosa & $0,46 \%$ \\
\hline C: úlcera vascular & $0,46 \%$ \\
\hline
\end{tabular}

Tabla 3. Diagnósticos relacionados con Sistemas.

\section{DISCUSIÓN}

Podemos entender que el servicio ofertado a los pacientes de Nefrología se corresponde, en mayor grado, con el rol autónomo de la enfermera, tal vez porque en gran medida el paciente de esta especialidad es un individuo con una enfermedad crónica con alto índice de comorbilidad y necesidades de cuidado. A unque el $34,70 \%$ de los cuidados interdependientes indica también la necesidad de observación de signos y síntomas ante posibles complicaciones, conllevando por ello cuidados del rol interdependiente.

Por otra parte, ante la alta prevalencia de diagnósticos de riesgo, podemos concluir que las actividades de prevención son un elemento fundamental entre los profesionales de enfermería, en el cuidado de los pacientes atendidos en la unidad de Nefrología, y el plan de cuidados establecido para cada paciente conlleva una serie de acciones cuidadoras planificadas por la enfermera, que definen su área de responsabilidad y dan respuesta a una necesidad específica de salud.

En la acción cuidadora demostramos la necesidad real de la aportación específica enfermera al sistema sanitario, donde, validando el rol independiente y el interdependiente, aseguramos la eficacia del ejercicio profesional.

Las enfermeras estamos demostrando ser cada vez más conscientes y responsables de la trascendencia de nuestra función. Es fundamental que identifiquemos qué hacemos, cómo lo hacemos, para quién lo hacemos y por qué son necesarios nuestros servicios, ya que así nos afianzaremos como profesión.

Este trabajo abre un campo de desarrollo profesional en los siguientes aspectos:

- Realización de sesiones de enfermería para profundizar en los resultados obtenidos.

- Reevaluación de los planes de cuidados estandarizados de la unidad que permitan trabajar en una enfermería basada en la evidencia, dando respuesta a las necesidades reales de nuestros clientes para contribuir a mejorar nuestra práctica clínica. 


\section{BIBLIOGRAFÍA}

1. Torres Torres LF, J urado Gámez Mâ j, Vera A costa J . Estudio descriptivo sobre la utilización del proceso de enfermería en una unidad de Hemodiálisis. Planes de cuidados estandarizados. Libro de comunicaciones XXII Congreso Nacional de la Sociedad Española de Enfermería Nefrológica. Santander 1997: 235-240.

2. Calvo Calvo MA. Diagnósticos enfermeros para la cartera de servicios de las unidades de trasplante renal. Libro de comunicaciones XXVI Congreso Nacional de la Sociedad Española de Enfermería Nefrológica. Zaragoza 2001: 135-141.

3. Luis MT, Fernández C, Navarro MV. De la teoría a la práctica. El pensamiento de Virginia Henderson en el siglo XXI. Masson; 1998.

4. Alfaro-LeFevre R. Aplicación del Proceso Enfermero. Springer; 1999.

5. Sastre H, López S. ¿Cuidados por actividades? ¿Cuidados por pacientes?. Rev ROL Enferm 1990; 141: 23-26.
6. Montesinos A. Dependencias de los enfermos y cargas de trabajo de Enfermería. Rev ROL Enferm 1988; 116: 43-45.

7. Luis M.T. Diagnósticos Enfermeros. Un instrumento para la práctica profesional. Mosby/ Doyma Libros; 1995.

8. Alberdi Castell R.Ma. Sobre las concepciones de la Enfermería. Rev ROL Enferm. 1988; 115: 25-30.

9. De Pedro J . ¿Existe el producto-servicio enfermero?. Revista Enfermería Clínica 1993; 3(6): 213-214.

10. Hernández Serra R.Ma . La medición del producto enfermero. Revista Enfermería Clínica 1995; 5(3): 111-118.

11. Del Pino Casado R, Ugalde Apalategui M. Líneas de investigación en diagnósticos enfermeros. Revista Enfermería Clínica 1999; 9(3): 115-120.

12.- Duarte Clíments $G$, Montesino A fonso N. El consenso como estrategia de implementación del diagnóstico enfermero, valoraciones estandarizadas y selección de etiquetas diagnósticas. Revista Enfermería Clínica 1999; 9(4): 142-151. 